\title{
Linear Block Equalizers in Rayleigh Fading Channel with Normalized Channel Impulse Response
}

\author{
Abhishek Kumar \\ Department of Electronics \& \\ Communication \\ SISTEC Bhopal India
}

\author{
Anoop Tiwari \\ Department of Electronics \& \\ Communication \\ SISTEC Bhopal India
}

\author{
Ravi Shankar Mishra \\ Department 4of Electronics \& \\ Communication \\ SISTEC Bhopal India
}

\begin{abstract}
Linear adaptive equalizers are widely used in wireless communication systems in order to reduce the effects of the channel distortion. Various researchers have used linear block equalizers for different modulations techniques. In this paper the BER performance of different M-PSK and M-QAM modulations with the block based LMS and RLS linear equalizers are compared over the flat and frequency selective Rayleigh fading channel. For achieving better performance the Rayleigh channel is modeled with four multipath channels and normalized channel impulse response under the presence of AWGN noise. The maximum Doppler shifts frequencies are varied for evaluating the performance of the equalizers. Using the normalized channel impulse response improves the BER performance of the communication system for the higher values of $M$ as 512 and 1024. Transmitted and received constellation diagrams are also compared for different equalizers. Performance is also compared for the different equalizer weights and block sizes.
\end{abstract}

\section{General Terms}

Adaptive Equalizers, Rayleigh fading channel, Wireless communication

\section{Keywords}

Linear Equalizers, LMS, RLS algorithm, Normalized channel impulse response, Bit error rate.

\section{INTRODUCTION}

The aim of equalizers is to reduce the fading effect, ISI, and noise from the multipath wireless networks and mobile communications channels. The equalizers may be broadly classified as linear and non-linear equalizer and used to ease the demodulation process at the wireless receivers. In wireless multipath communication Rayleigh fading is a common phenomenon which exist due to reflection of waves, diffraction, and scattering of the transmitted waves from the large physical structures such as buildings or mountains as shown in Figure 1. Since with the increasing number of wireless users the higher size modulation methods are frequently adopted thus it needs to compare the performance of modulation techniques over fading environment. In the current work performance of various linear equalizers are evaluated over the flat fading and frequency selective fading channels.

The Least mean square (LMS) equalizers have been widely used in literature [1,2, and 4]. But due to its linear nature these equalizers fails when BER performance go beyond $1 \%$ limit. These equalizers also give lower mean square error (MSE). The linear and non-linear equalizers with Least Mean Squares (LMS) algorithm have been compared with the equalizers using the Recursive Least Mean Squares (RLS) algorithms. The LMS method has a low complexity and it is globally convergent if the desired values are given correctly. If the desired symbols are not correct, it does not converge. RLS algorithm has better convergence characteristics than the LMS algorithm. But, it has higher computational complexity than LMS algorithm. The general RLS algorithm's complexity grows with $\mathrm{N}^{2}$ where $\mathrm{N}$ is the number of equalizer coefficients.

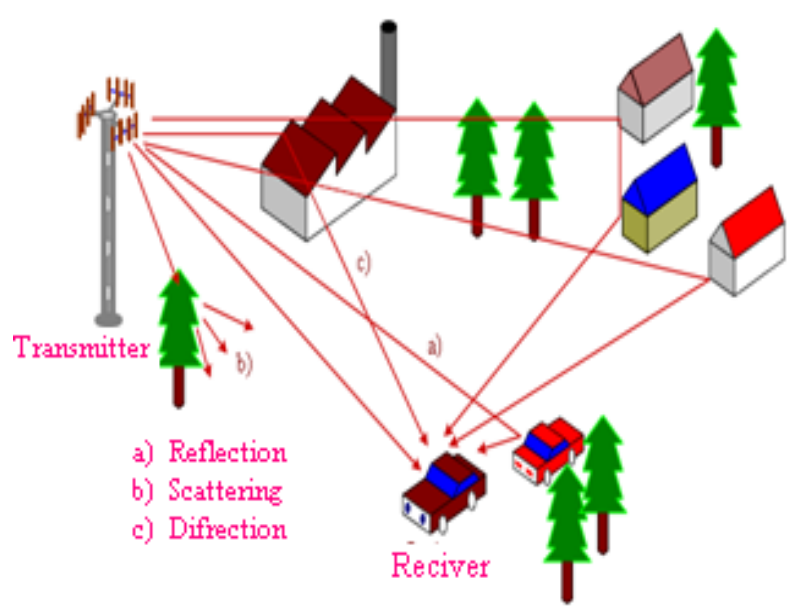

Figure 1 Reasons of Fading in Multipath wireless channels

There are also RLS algorithms that have computational complexities which grow linearly with the increasing number of equalizer coefficients. These algorithms are called fast RLS algorithms [1, and 4]. The main advantages of equalizing the received signal before demodulation techniques are i) reduction of Inter symbol Interference (ISI), ii) reduction of Inter Carrier Interference (ICI), ii) reduction of noise. But every equalizer performs differently under different working environments. Therefore, it is desired to identify and analyze the performance of linear equalizers over different modulation techniques such as PSK and QAM

In this paper a comparative analysis of LMS and RLS equalizers with different M-PSK and M-QAM modulations over the Rayleigh fading channels are presented. In order to compare the performance various equalizer parameters are varied and Bit error rate (BER) is evaluated and compared. In this paper after introduction section 2 discusses brief literature review of the existing work followed by the various issues in multipath propagation (such as fading and interference) in section 3 . The proposed communication system block diagram is explained in the section 4. The LMS and RLS linear equalizers are described in the section 5. The results of performance evaluation are given in the section 6 followed by the conclusion in section 7 . 


\section{LITERATURE REVIEW}

The equalizers are widely used by the researchers for reducing the channel distortions. The most commonly used algorithm from the performance and complexity point of view is the LMS algorithm [2]. The LMS algorithm based equalizer is not effective when the desired symbols are incorrect such as under the noisy channel,

Alireza et al. [2] have proposed a blind linear equalization, and data detection, for an efficient, and un-coded transmission over a frequency selective Rayleigh fading channel. The method uses the LMS algorithm for their analysis. The maximum Doppler shift is taken as $20 \mathrm{~Hz}$. and SNR is varied from 0-20. dB. Wing et al. [5] have presented the method for equalization of linear frequency selective fading channel which reduces the effect of inter-symbol interference. The method optimizes transmitter and receivers filters impulse response to reduce the inter symbol interferences.

Sabita et al. [4] have presented a comparative analysis of different modulation schemes is performed in a fading environment using the adaptive equalization technique for the mitigation of fading distortion. The comparison is made at a fixed SNR of $35 \mathrm{~dB}$. They have concluded that QAM performs better than QPSK technique. Many researchers have adapted the variable Tap length for improving the equalizer performances [7, and 8]. Yu Gong et al. [6] have proposed a MMSE equalizer which jointly adopts the tap length and decision delay for improving the performance. But method was computationally complex. Kiran Kuchi [9] has presented the performance comparison of the zero forcing ( $\mathrm{ZF}$ ) and MMSE linear equalizers under multi antenna Rayleigh fading channel system.

X. Ma and W. Zhang, [10] have explained the basic fundamental limitations of the linear equalizers: such as capacity, diversity, and computation complexity. Jaymin et al. [11] have presented a comparative analysis of the MLSE, LMS and RLS non linear adaptive equalization algorithms for the wireless digital communication. Each algorithm is tested for the BPSK, 4PSK and 16QAM modulation techniques.

Fu Shaozhong et al.[12] have updated the length of LMS equalizer for using exponential function. Method reduces the average number of iterations and thus converges faster than standard LMS algorithm. Veeraruna et al. [13] have analyzed the performance of LMS linear equalizer in the decision directed mode over the fading channel. The equalizer is approximated by the one dimensional differential equation (ODE) but method seems slightly complex. Garima Malik et al. [14] have given a brief overview of the RLS and LMS adaptive equalizers. They have concluded that bandwidth efficient communication is possible by compensating the time varying channel distortions using equalizers.

But the performance of different M-PSK and M-QAM modulation techniques over the fading channels is not yet evaluated for different adaptive equalizers. Also it is needed to evaluate the performance of linear equalizers for different velocities corresponding to different maximum Doppler shifts of the fading channel. These are the prime goals of this paper.

\section{CHANNEL DEGRADATION AND CHANNEL MODEL}

The wireless radio channels are greatly affected by the structures in their surrounding such as mountains, cars or buildings. These structures cause the multipath reflections of the transmitted signal and attenuate the transmitted power, called fading. This is the major reason of signal degradation in the wireless communication. There are many types of fading channels. Rayleigh fading channel is most commonly used.

\subsection{Rayleigh Fading Model}

Rayleigh fading channel is a statistical model which assumes that the power of a signal that has passed through transmission medium varies randomly, or faded as per the Rayleigh distribution. It is mostly applicable if there is no line of sight propagation exists between the transmitter and receiver. Or in other words Rayleigh fading occurs in the dense area between transmitter and receiver which only have indirect path called NLOS to receive the resultant waves. The Rayleigh fading models are of two basic types as: Flat or Frequency selective.

\subsubsection{Flat Fading (Non-Selective Fading)}

Flat fading channel is a kind of fading in which all available frequency components of the received signal varies simultaneously at the same ratio [5] .The flat fading occurs when delay spread spectrum of the signal at the receiver is less than the channel bandwidth and symbol period.

\subsubsection{Frequency Selective Fading}

Frequency selective fading is a time varying fading which unequally affects the different spectral components of the radio signal [5]. This fading occurs when the channel bandwidth and delay spread spectrum of a signal at the receiver is higher than the channel bandwidth and symbol period. The fading channel is modelled with a linear and timevarying Channel Impulse Response (CIR) denoted by the function $h(t, \tau)$.

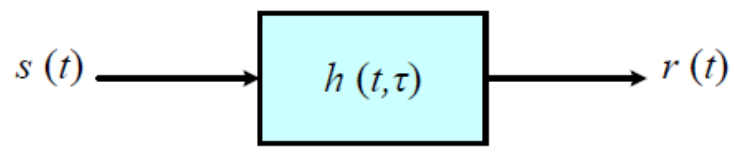

Figure 2 Modelling fading channel

$$
r(t)=h(t, \tau) * s(t)
$$

In discrete time the channel is modeled as impulse response of the channel filter for $\mathrm{n}^{\text {th }}$ samples;

$$
r(n)=\sum_{i=0}^{N} C_{i} s(n-i)
$$

Where, $\mathrm{s}(\mathrm{n})$ is the input signal,

$$
\begin{aligned}
& r(n) \text { is the output signal, and } \\
& C_{i} \text { is the filter coefficients. }
\end{aligned}
$$

Where, $\mathrm{N}$ is known as the filter order; an Nth-order filter has $(\mathrm{N}+1)$ terms on the right-hand side; these are commonly referred to as taps.

\subsection{Channel Normalization}

In order to improve the PSK performance for number of phases, in this paper it is proposed to use the normalized channel impulse response with respect to maximum absolute response value as:

$$
\begin{gathered}
M x=\max (\max (|r(n)|)) \\
r_{\text {norm }}(n)=\frac{r(n)}{M x}
\end{gathered}
$$

The normalized impulse response is added with the additive white Gaussian noise $v(n)$ the noisy channel response is given as:

$$
\text { chan }=r_{\text {norm }}(n)+v(n)
$$




\section{PROPOSED METHOD}

The proposed method uses Linear equalizers in a training mode operation and use of the Nyquist Pulse shaping filters efficiently minimizes the inter symbol interference (ISI) induced by the channel. The block diagram of the proposed wireless communication method is shown in Figure 3.

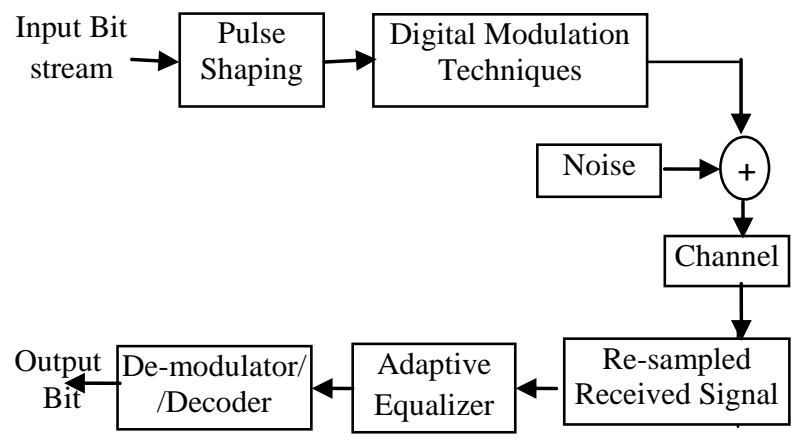

Figure 3 Block diagram of proposed wireless communication system

As another experiment the BER for different Doppler Shift frequencies are compared for 256 PSK and RLS equalizer over the frequency selective fading channel as shown in Figure 9. The equalized signals are demodulated to reconstruct the desired bit sequences. The input bit sequences are first modulated using M-PSK or M-QAM modulation techniques and then square root raised cosine filters are used as pulse shaping filters. The channel is modeled as Rayleigh flat fading channel. The channel impulse response is normalized using the maximum response value. Then linear equalizers are implemented at the receiver to reduce the channel distortions.

\section{LINEAR EQUALIZERS}

An adaptive equalizer is a filter that automatically adapts to time-varying properties of the wireless channel [16]. In this paper the equalizer is designed in a training mode. It can be implemented by performing the tap weight adjustments periodically or continually. These periodic adjustments are accomplished by periodically transmitting a preamble or short training sequence of digital data known by the receiver. Continual adjustment are accomplished by replacing the known training sequence with a sequence of data symbols estimated from equalizer output and treated as known data.

Architecture of the adaptive equalizer is shown in the Figure 4 below. The coefficients of the filters are called as weights of the system and are updated according to the type of equalizer algorithm. The equalizer output is calculated as;

$$
y(n)=\sum_{k=0}^{M-1} u(n-k) w_{k}^{*}(n)
$$

The error signal is calculated as;

$$
e(n)=d(n)-y(n)
$$

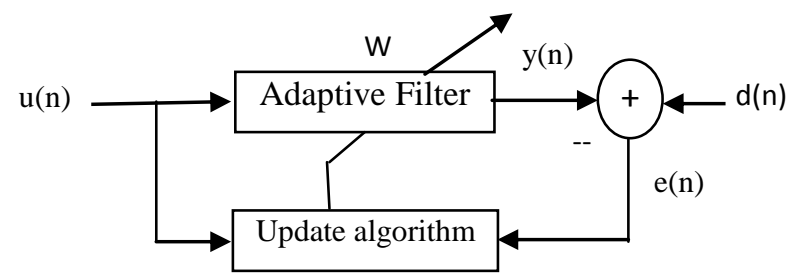

Figure 4 Adaptive Equalizer

Where, w: weight vector or coefficients

$$
\begin{aligned}
& \text { u: Input vector } \\
& \text { e: error signal }
\end{aligned}
$$

In order to minimize the error signal, the weights are updated using either LMS or RLS algorithms.

\subsection{Standard LMS Algorithm}

The standard least mean squares (LMS) algorithm is a type of adaptive filter which adapts the filter coefficients to produce the least mean squares error signal between the desired and the actual signal. LMS is a stochastic gradient descent method in which the filter is adapted based on the error at the current time. LMS filter is built around a transversal (i.e. tapped delay line) structure. The LMS filter or equalizers employ the small step size $\mu$ which provides a simple and accurate representation of the filter transient behavior. Let $\mathrm{w}$ is the initial weight then;

$$
G=\text { ? } * u(n-k)
$$

Updated weights are calculated as;

$$
w_{k}(n+1)=L_{\text {factor }} * w_{k}(n)+G * e^{*}(n)
$$

Where, $L_{\text {factor }}$ is the leakage factor which is 1 for standard LMS algorithm..

\subsection{Standard RLS Algorithm}

The Recursive least squares (RLS) [11, 14 and 17] based adaptive filter algorithm is which recursively finds the filter coefficients and minimize the weighted linear least squares cost function associated to the input signals. RLS algorithm converges faster than other methods but is computationally complex. The structure of the RLS algorithm is similar to that of LMS as in Figure 4. The RLS algorithm uses covariance matrix updating formula [14], which is used for automatics adjustment corresponding to the estimation error. The initial inverse correlation matrix Delta is given as;

$$
\text { Delta }=\delta^{-1} I
$$

Where, Delta is the inverse correlation matrix and $\square[$ is regularization parameter which is positive constant for high SNR and negative for the low SNR value. For any time instance $\mathrm{n}=1,2,3 \ldots$.

$$
\begin{gathered}
x(n)=\text { Delta } * u(n-k) \\
G(n)=\frac{x(n)}{\lambda+u^{T}(n) * x(n)}
\end{gathered}
$$

Where, $\lambda=$ Forget Factor;

The updated value of inverse correlation matrix is given as;

$$
\text { Delta }=1 / \lambda *\left(\text { Delta }-G(n) * u^{T}(n) * \text { Delta }\right)
$$


The updated weights are calculated as;

$$
w_{k}(n+1)=w_{k}(n)+G(n) * e^{*}(n)
$$

The RLS algorithm and LMS algorithm are opposite of each other. The LMS algorithm updates faster but converges slower, on the other hand RLS algorithm converges faster but updates slower.

\section{EXPERIMENTAL RESULTS}

In this paper the Bit error rate (BER) of the various M-PSK and M-QAM modulation techniques are compared for different linear adaptive block equalizers. The simulation is performed on MATLAB software. Every transmitting block contains payload of length 400. Training and Tail sequences are appended before and after the payload data respectively. The various input parameters used for the simulation are given in the Table 1. In the paper the channel is modeled with normalized Channel Impulse Response (CIR). The Figure 5 compares the BER performance of the LMS and RLS linear equalizers respectively for QPSK modulation. In each simulation average BER of 50 blocks is plotted.

Table 1 Used input parameters

\begin{tabular}{|l|c|l|}
\hline Variable & Value & \multicolumn{1}{c|}{ Description } \\
\hline Nbitpsk & $2-10$ & Bits per PSK Symbol \\
\hline Ts & $1 \mathrm{e}-6$ & Sampling time \\
\hline $\mathrm{M}$ & $4-1024$ & Size of Modulation \\
\hline NTap & 4 & Length of equalizer \\
\hline xPayload & randi $(1,400)$ & $\begin{array}{l}\text { Number of data bits per } \\
\text { block }\end{array}$ \\
\hline EbNo & 0 to 20 & Range of SNR \\
\hline Fd & $30 \mathrm{~Hz}$ & Doppler Shift \\
\hline Chan & Channel & Rayleigh fading channel \\
\hline osfFilt & 4 & Over sampling factor \\
\hline D & $1 \mathrm{e}-6[04812]$ & Multipath Delay vector \\
\hline G & {$[0 \quad 69]$ dB } & Multipath Gain vector \\
\hline nBlocks & 50 & Number of blocks \\
\hline Step & 0.1 & LMS Step size \\
\hline Forgot & 0.9 & RLS Forgot Factor \\
\hline
\end{tabular}

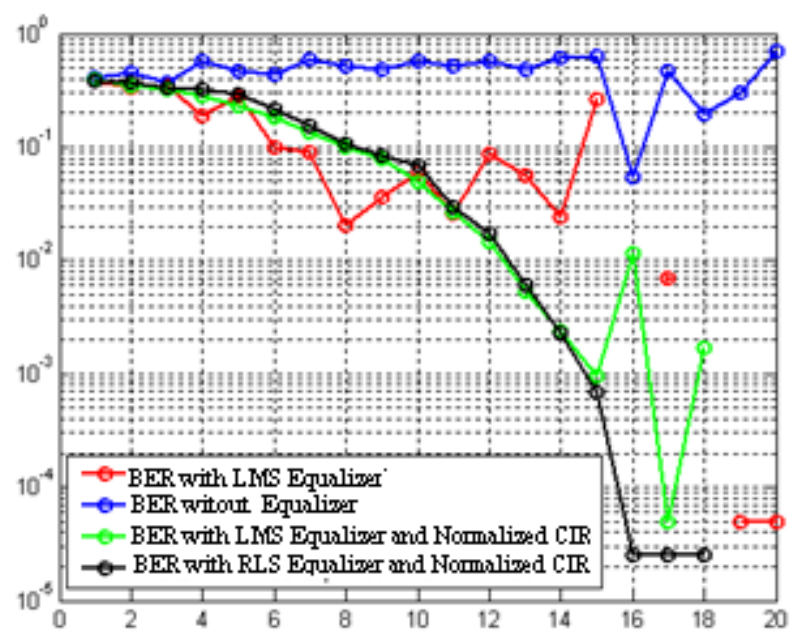

Figure 5 BER for LMS and RLS equalizers for frequency selective fading channel with step size 0.1 and forgot factor 0.9

It is found that using the normalized CIR improves the performance of linear equalizers significantly. It is found that
LMS equalizers can achieve minimum BER order of $10^{-4}$ as in Figure 5, while RLS equalizer can achieve better BER performance of around $10^{-5}$ range at the higher SNR of around $18 \mathrm{~dB}$ as can be observed from Figure 5. Channel Normalization produces the smooth BER curves and also reduces the error probability.

The BER performance of the different M-PSK with $\mathrm{M}=4$, 16, 256, 512 and 1024 are, compared for RLS equalizer under the flat and frequency selective fading channels respectively in Figure 6 and Figure 7. It can be observed that up to around $16 \mathrm{~dB}$ the proposed system with normalized CIR performs approximately similar for all PSK sizes. It can be also observed that proposed method of normalized channel impulse response performs better for even PSK size of up to 512 and 1024. In the Figure 8 the comparison of BER performance of the different M-QAM with $\mathrm{M}=16,256,512$ and 1024 are, compared for RLS equalizer under the flat and frequency selective fading channels. It is found that QAM gives poor BER performance but since there is not much variation in BER at higher QAM size so it is widely used. The BER performance may be improved by using channel coding methods.

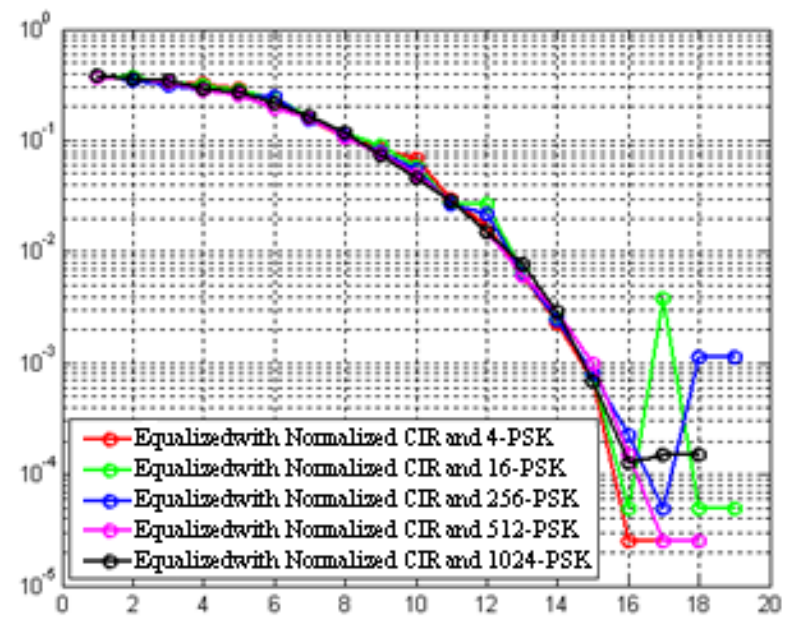

Figure 6 BER Comparison for M-PSK modulation techniques for RLS equalizer with flat Fading normalized CIR.

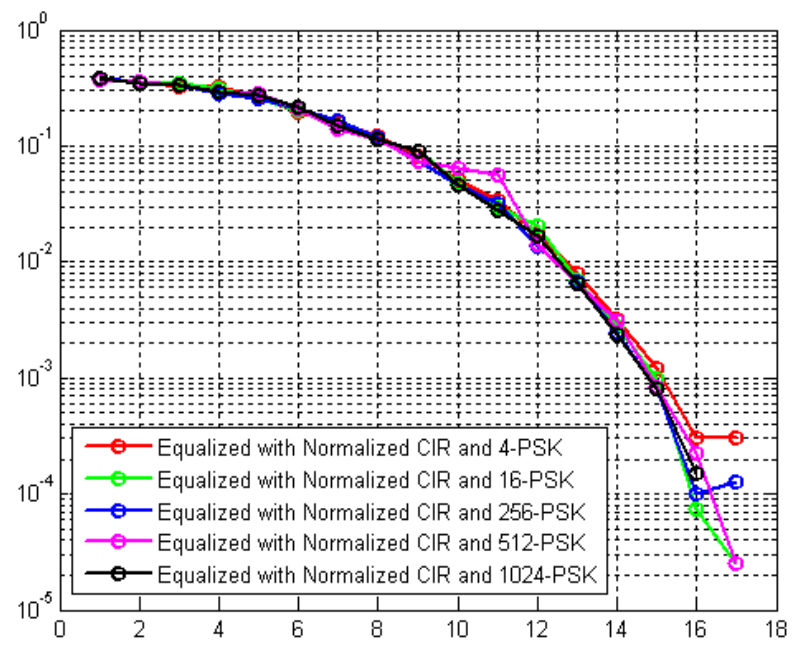

Figure 7 BER Comparison for-PSK modulation techniques for RLS equalizer with Frequency Selective Fading normalized CIR. 


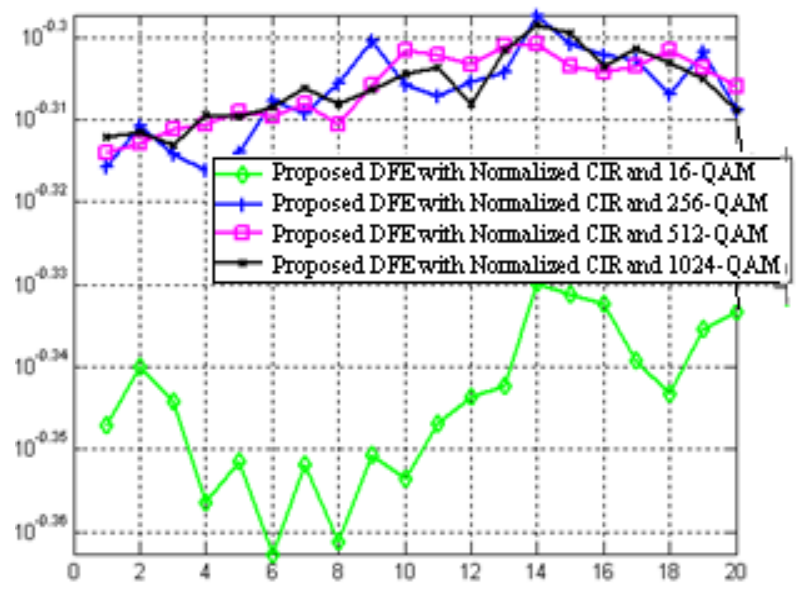

Figure 8 BER Comparison for M-QAM modulation techniques for RLS equalizer with frequency selective fading normalized CIR.

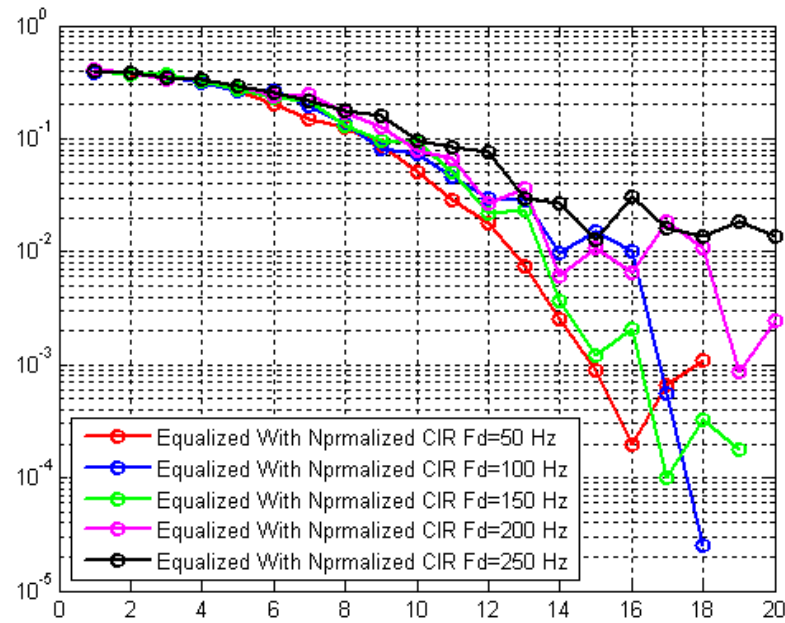

Figure 9 BER for 256 PSK modulation techniques for RLS equalizer with Frequency Selective Fading normalized CIR

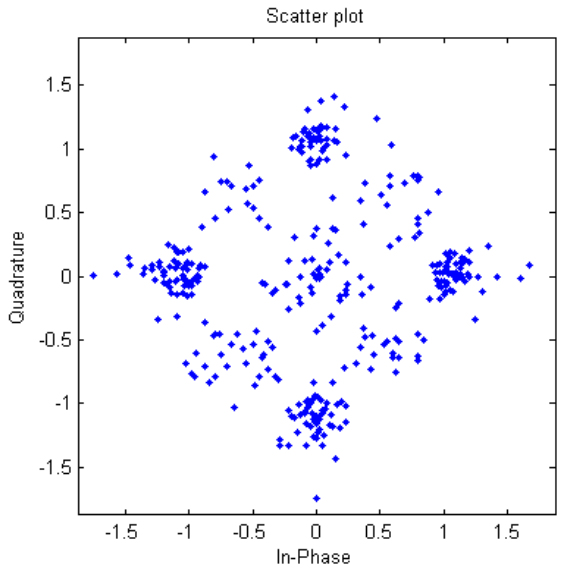

a) Transmitted signal Scatter plot

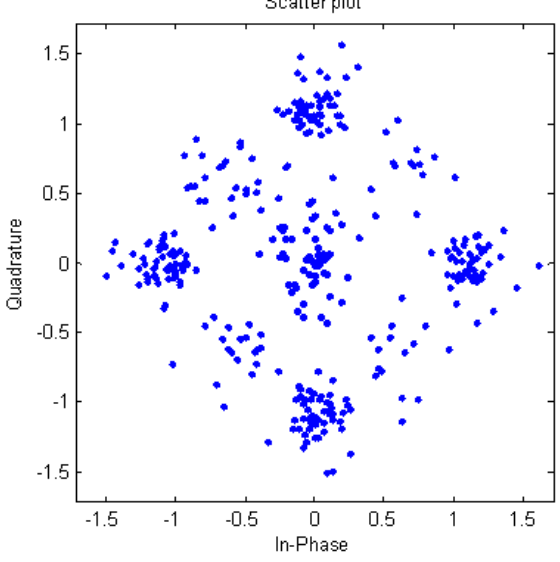

a) Transmitted signal

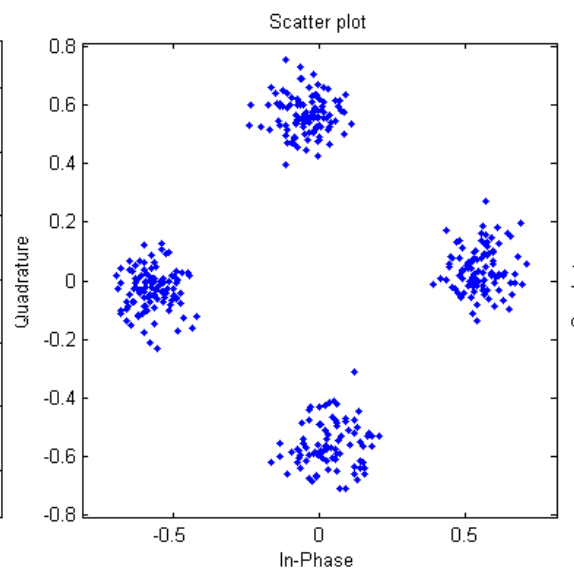

b) Received with Flat fading channel

Scatter plot

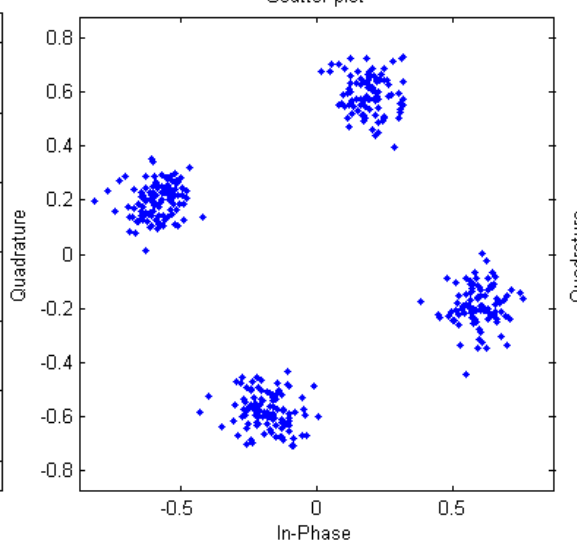

b) Received with Frequency selective fading channel

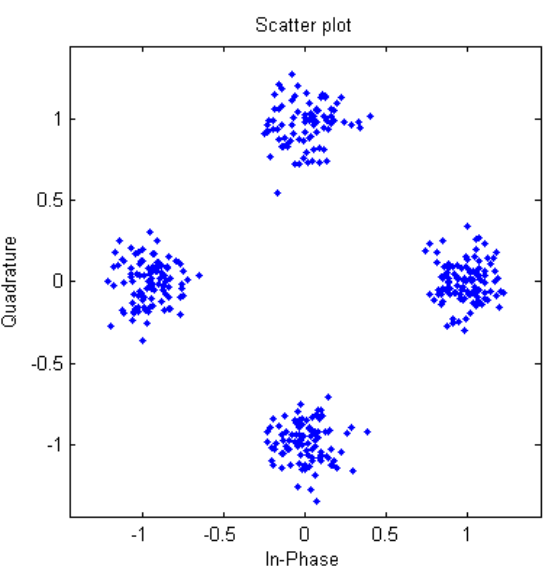

c) Equalized with RLS equalizer

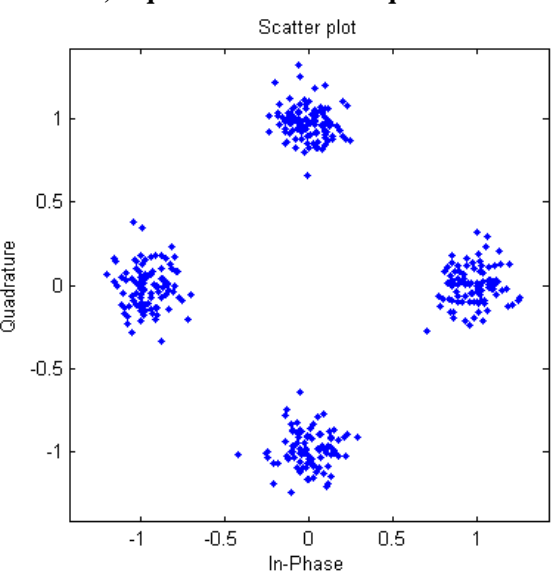

c) Equalized with RLS equalizer

Figure 10 Constellation diagram of the QPSK modulation for flat and frequency selective fading channels

As another experiment the BER for different Doppler Shift frequencies are compared for 256 PSK and RLS equalizer over the frequency selective fading channel as shown in Figure 9. The maximum Doppler shift frequency is varied as $50,100,150,200$, and $250 \mathrm{~Hz}$. It is found that the significant BER can be achieved even at $200 \mathrm{~Hz}$ Doppler frequency although the BER performance degrades with the increase in Doppler shift frequency.

The Figure 10 presents the comparison of the constellation diagram of the QPSK modulation with flat and frequency selective channels for transmitted, received and equalized 
sequences. It is clear that more scattered pattern is there under multipath fading frequency selective channel.

\section{CONCLUSION}

The paper proposes the new method of Rayleigh fading channel model using normalized channel impulse response. The performance of block based linear LMS and RLS equalizers are compared for different M-PSK and M-QAM modulations. It is found that QAM gives poor BER performance than PSK. It is observed that modeling channel with normalized channel impulse response significantly improves the BER performance of the PSK modulation at higher PSK sizes of 512 and 1024 receptively. It is found that the RLS equalizer gives better performance than LMS in terms of minimum BER. The linear block equalizers are widely used because of its simplicity. These are useful where channel parameter does not vary frequently. But the complexity increases linearly with the increase in tap weights. The performance is also compared for the different Doppler shift frequencies corresponding to different user velocities. It is found that BER performance degrades with the increase in Doppler shift. In future decision feedback equalizers and channel coding may be implemented for the proposed communication system for further improving the BER performance.

\section{ACKNOWLEDGMENTS}

I would like to thanks my guide Mr.Anoop Tiwari and Dr. Ravi Shankar Mishra for their technical support and guidance for this work. I would also like to thanks my family members for their support

\section{REFERENCES}

[1] S C Lin, "Performance analysis of decision feedback equalizer for cellular mobile radio co-channel interference and fading" IET Communicatio Vol. 3, Issue. 1, pp. 100-114 2009,

[2] S. Alireza Banani, Rodney G. Vaughan, "Itterative Blind Linear Equalizer in time varying disapersive channel", $3^{\text {rd }}$ International conf. on Electrical and Computer Engineering (CCECE) pp 1-6 2010.

[3] Xin Wang and Guangzeng Feng. "A constant Modulous algorithm for phase modulation signal", IEEE $7^{\text {th }}$ international Conf. on Networking, pp. 584-587, 2008

[4] Sabita Nahata, 2subrata Bhattacharya, "Comparative analysis of modulation schemes using Adaptive Equalizers as a fading mitigation technique", International Journal of Electronics Signals and Systems, Vol-1 Iss-3, pp. 25-31, 2012

[5] Wing Seng Leon,, Umberto Mengali, "Equalization of Linearly Frequency-Selective Fading Channels", Ieee Transactions On Communications, Vol. 45, No. 12, December 1997

[6] Yu Gong, Xia Hong and Khalid F. Abu-Salim, :l" Adaptive MMSE equalizer with optimum Tap length and Decision delay", IEE 2010

[7] F. Riera-Palou, J. M. Noras, and D. G. M. Cruickshank, "Linear equalisers with dynamic and automatic length selection," Electronic Letters, voL 37, no. 25, pp. 1553 1554, Dec. 2001

[8] Y. Gu, K. Tang, H. Cui, and W. Du, "LMS algorithm with gradient descent filter length," IEEE Signal
Processing letters, voL II, no. 3, pp. 305 - 307, March 2004.

[9] Kiran Kuch, "Limiting Behavior of ZF/MMSE Linear Equalizers in Wideband Channels with Frequency Selective Fading", IEEE Communications Letters, Vol 16, No. 6, June 2012

[10] X. Ma and W. Zhang, "Fundamental limits of linear equalizers: diversity, capacity, and complexity," IEEE Trans. Inf. Theory, vol. 54, pp. 3442-3456, Aug. 2008.

[11] Jaymin Bhalani, A.I.Trivedi, Y.P.Kosta , "Performance Comparison of Non-Linear and Adaptive Equalization Algorithms for wireless communication channel" IEEE 2009

[12] Fu Shaozhong, Ge Jianhua $\square$ Wang Yong, “ Fast adaptive algorithm for variable length equalizer based on exponential", Proc. of IEEE International Conferences o Wireless Communication, Networking and Mobile Computing.WiCOM 08, 2008

[13] Veeraruna Kavitha and Vinod Sharma, "Tracking performance of LMS-iinear equalizers for fading channel", Forty-Fourth Annual Allerton Conference Allerton House, UIUC, Illinois, USA, pp. 681-686, Sept 2006.

[14] Garima Malik, Amandeep Singh Sappal, “Adaptive equalization algorithm: An Overview", International Journal of Advanced Computer Science and Applications (IJACSA) Vol. 2, No.3, March 2011

[15] Wee-Peng Ang, B. Farhang-Boroujeny, "A New Class of Gradient Adaptive Step-Size LMS Algorithms", IEEE Trans. On Signal Processing, Vol. 49, No. 4, April 2001

[16] A. Molisch, "Wireless Communication", E- Book Wiley-IEEE Press in 2011

[17] Suneeta V. Budihal, Priyatamkumar, R.M.Banakar, "Performance analysis of Adaptive Decision Feedback Turbo Equalization (ADFTE) using Recursive Least Square (RLS) Algorithm over Least Mean Square (LMS) Algorithm", IEEE International Conference on Computational Intelligence and Multimedia Applications 2007

\section{AUTHORS}

1. Abhishek Kumar: Have received his B.Tech degree in Electronics \& Telecommunication Engineering in 2008 from G.H.I.T.M, Puri (Orissa), India. He has three years of teaching experience as a lecturer in Cambridge Institute of Technology, Ranchi (Jharkhand) and currently he is pursuing his M. Tech. degree in Digital Communication from Sagar Institute of Science \& Technology, Bhopal (Madhya Pradesh).

2. Prof. Anoop Tiwari: $\mathrm{He}$ is working as an Assistant Professor in the department of Electronics \& communication engineering in Sagar Institute of Science \& Technology, Bhopal

3. Dr. Ravi Shankar Mishra: Have received $\mathrm{PhD}$ degree in the VLSI field from MANIT Bhopal, and is currently working as Head of the department ECE, SISTEC Bhopal, India. 